\title{
Thermal manipulation of plasmons in atomically thin films
}

\author{
Eduardo J. C. Dias (10)', Renwen Yu and F. Javier García de Abajo (1) ${ }^{1,2}$
}

\begin{abstract}
Nanoscale photothermal effects enable important applications in cancer therapy, imaging and catalysis. These effects also induce substantial changes in the optical response experienced by the probing light, thus suggesting their application in all-optical modulation. Here, we demonstrate the ability of graphene, thin metal films, and graphene/ metal hybrid systems to undergo photothermal optical modulation with depths as large as $>70 \%$ over a wide spectral range extending from the visible to the terahertz frequency domains. We envision the use of ultrafast pump laser pulses to raise the electron temperature of graphene during a picosecond timescale in which its mid-infrared plasmon resonances undergo dramatic shifts and broadenings, while visible and near-infrared plasmons in the neighboring metal films are severely attenuated by the presence of hot graphene electrons. Our study opens a promising avenue toward the active photothermal manipulation of the optical response in atomically thin materials with potential applications in ultrafast light modulation.
\end{abstract}

\section{Introduction}

Heat generation driven by light absorption in nanostructures has proven useful for photothermal therapy ${ }^{1-3}$, nanoscale imaging ${ }^{4,5}$, data storage ${ }^{6}$, photocatalysis $^{7}$ and photodetection $^{8,9}$. These important applications have been extensively investigated in conducting materials due to the substantial enhancement produced in the strength of nanoscale photothermal processes as a result of the excitation of their collective electron oscillations, known as plasmons ${ }^{10-12}$. Among plasmonic materials, graphene offers additional appealing properties, such as large field confinement and enhancement ${ }^{13-15}$, remarkably low optical losses ${ }^{14,16}$ and the ability to tune its plasmons electrically $^{17-27}$, which have generated expectations for applications in optical modulation ${ }^{23,28-31}$, light detection $^{8,17,22,32,33}$, and sensing ${ }^{34-36}$. Unfortunately, plasmons in graphene have been observed only in the mid-infrared

Correspondence: Renwen Yu (renwen.yu@icloud.com) or F

Javier García de Abajo (javier.garciadeabajo@nanophotonics.es)

'ICFO-Institut de Ciencies Fotoniques, The Barcelona Institute of Science and Technology, 08860 Castelldefels (Barcelona), Spain

${ }^{2}$ ICREA-Institució Catalana de Recerca i Estudis Avançats, Passeig Lluís

Companys 23, 08010 Barcelona, Spain
(mid-IR) spectral range, far from the technologically appealing visible and near-infrared (vis-NIR) spectral regime. Photothermal excitation has been proposed as a way to reach the vis-NIR plasmonic regime ${ }^{37}$, but experimental attempts in this direction have only been limited to the mid- $\mathrm{IR}^{38,39}$. As an alternative approach to extrapolate the appealing plasmonic properties of graphene to vis-NIR, both monolayer noble metals ${ }^{40}$ and hybrid systems formed by graphene in close proximity to few-nanometer metal films ${ }^{41}$ have been predicted to display relatively low losses and a large electrical tunability in the vis-NIR domain. Progress in this direction has been recently made with the experimental demonstration of plasmons in laterally patterned sub-2-nm crystalline silver $^{42}$ and thicker amorphous gold ${ }^{43}$ films. In particular, epitaxially grown silver films serve as a novel platform for ultracompact nanophotonic devices that can further benefit from the comparatively high quality factor of plasmons in defect-free crystalline silver samples ${ }^{42}$.

Under ultrafast light irradiation, the absorbed energy is first deposited on conduction electrons, which remain at an elevated temperature for $\sim 1$ ps before transferring a substantial fraction of heat to the atomic lattice ${ }^{44}$. 
Importantly, due to the small electronic heat capacities of graphene $\mathrm{e}^{37,45}$ and ultrathin noble metals ${ }^{46}$ compared with their total heat capacities, the lattice remains close to ambient temperature for optical pump-pulse fluences as high as $\sim 10 \mathrm{~J} / \mathrm{m}^{2}$, which is the upper limit of the range typically used to investigate thin-film dynamics through pump-probe spectroscopy ${ }^{47,48}$. In combination with the strong temperature dependence of the graphene optical conductivity, the transmission and reflection of a light probe can undergo radical variations produced by heating $^{49}$, while intense photothermal effects should be expected due to the exceptionally small electronic heat capacity and generally weak electron-phonon coupling in this material ${ }^{45}$. Metals present a weaker thermal dependence, although the effects of intense pumping have also been observed to affect the probe as heat propagates away from the pumped region ${ }^{48}$. Consequently, graphene, thin metal films and hybrid systems comprising these materials hold strong potential to achieve large photothermal modulation over a broad spectral range extending from the visible to the $\mathrm{THz}$ region. Despite the vast range of applications that such modulation could open, the ultrafast photothermal response of these systems has been poorly explored.

In this paper, we theoretically investigate the thermal control of plasmons assisted by light absorption in graphene, thin metals and graphene/metal hybrid films. Based on a realistic description of the temperaturedependent optical properties of these materials, combined with the light-electron-lattice heat flow under ultrafast optical pumping, we predict a remarkable thermally driven attenuation of the plasmon strength in mid-IR graphene plasmons and vis-NIR metal plasmons of hybrid systems. This attenuation is directly revealed by plotting the reflection coefficient of planar structures. We further propose a practical scheme to exploit this dependence, consisting of laterally patterning the metal so that propagating external light can directly couple to the noted plasmons. Our results predict a large modulation of the light reflection produced by optical pumping using attainable intensities below the damage threshold level. Graphene plays an important role in these structures because in its absence the photothermal response is much weaker; we relate this result to the small heat capacity of the carbon layer. Our findings open a promising route towards ultrafast photothermal light modulation over a broad spectral range extending from vis-NIR to far-IR.

\section{Results}

We illustrate the main concept explored in this paper in Fig. 1a. Although the manipulation of the graphene conductivity is possible through electrical gating or chemical doping, these methods cannot reach the ultrafast regime that might become necessary for future applications. We need to resort to intrinsically ultrafast approaches, such as optical pump-probe techniques. In the present work, we consider that an optical pump heats the electrons of a part of a structure, causing large changes in the optical response of the entire system, which in turn translate into frequency and intensity modulations of its optical resonances. We apply this idea to graphene, few-monolayer noble metal films and graphene/metal hybrid systems (Fig. 1b). Before analyzing the optical modes of these structures, it is useful to review the photothermal properties of their constituents, chosen to be among the best currently available plasmonic materials.

\section{Thermoplasmonic properties of atomically thin films}

Graphene displays stronger photothermal effects than noble metals (see Fig. 1c) due to its conical band structure, in contrast to the free-electron-like behavior of the latter. A small amount of heat deposited on graphene electrons causes large temperature variations and changes in its optical response because the electronic heat capacity of this material is remarkably small ${ }^{37}$. This effect is thus an ideal ingredient for the pump-probe scenario envisioned in Fig. 1a. We anticipate that the strong graphene photothermal response can be exploited to modulate (visNIR) metal-like and $(\mathrm{THz})$ acoustic plasmons in graphene/metal hybrid systems (Fig. 1b). The electronic heat capacity of graphene increases with the Fermi energy $E_{\mathrm{F}}$ (i.e., by injecting more electrons in the system, see Fig. 1c), but it still remains orders of magnitude lower than the lattice heat capacity $\left(\sim 350 \mathrm{JK}^{-1} \mathrm{~m}^{-1}\right.$ at room temperature $^{50}$ ) for realistic levels of doping, and the same is true for noble metals. A femtosecond optical pump can then raise the electron temperature $T_{\mathrm{e}}$ to $1000^{\prime} \mathrm{s} \mathrm{K}$, while heat transfer to the lattice, which takes place over a picosecond timescale $^{44}$, does not substantially move the lattice temperature $T_{\ell}$ away from the ambient level $T_{0}$. In fact, we estimate that for fluences of the order of $1-5 \mathrm{~mJ} / \mathrm{m}^{2}$, which roughly correspond to ultrafast pulses of $\sim 100 \mathrm{fs}$ with peak intensities of $0.1-0.5 \mathrm{GW} / \mathrm{cm}^{2}$, the electron temperature in graphene increases up to $\sim 5000 \mathrm{~K}^{51,52}$, whereas electrons in the metal stay within a few 100 's K, as shown in Fig. S1 in the Supplementary Information (SI). This finding is a direct consequence of the much lower electronic heat capacity in graphene than in metals, as shown in Fig. 1c. We stress that the conditions described above are well below the reported damage threshold of graphene ${ }^{53}$. Therefore, in what follows, unless otherwise stated, we assume $T_{\ell}=T_{0}=300 \mathrm{~K}$ in both graphene and metal, which is a good approximation under the conditions of typical pump-probe experiments during a short time after pumping.

The optical response of graphene is highly dependent on $T_{\mathrm{e}}$ through the chemical potential $\mu$, the Drude weight $\mu^{\mathrm{D}}$ and the inelastic scattering rate $1 / \tau$, which enter its 
a

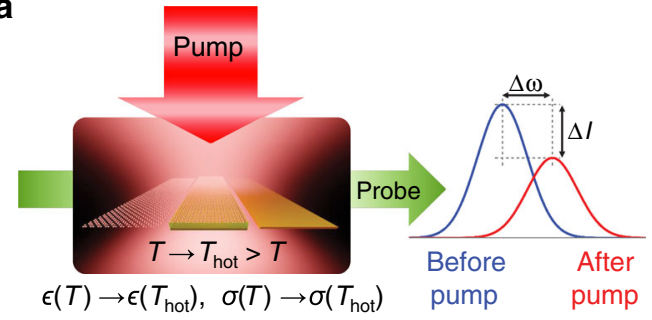

c

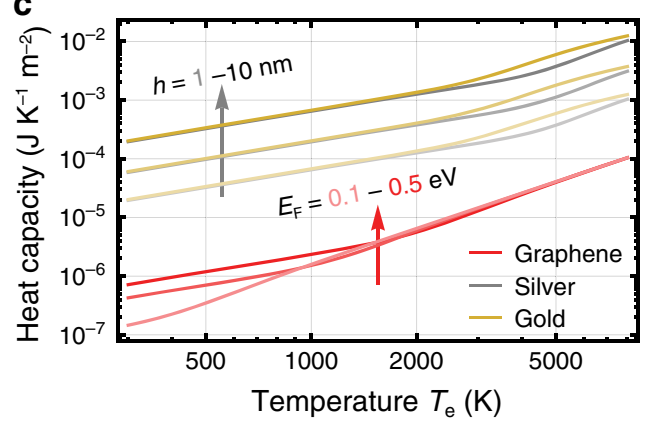

e

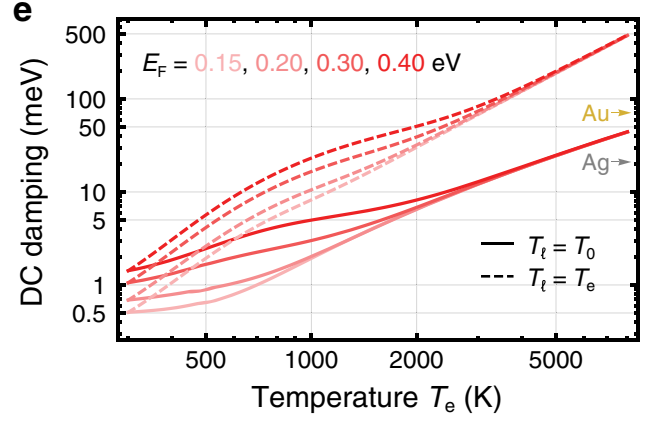

b

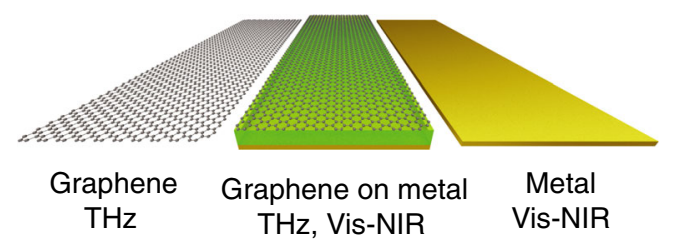

d

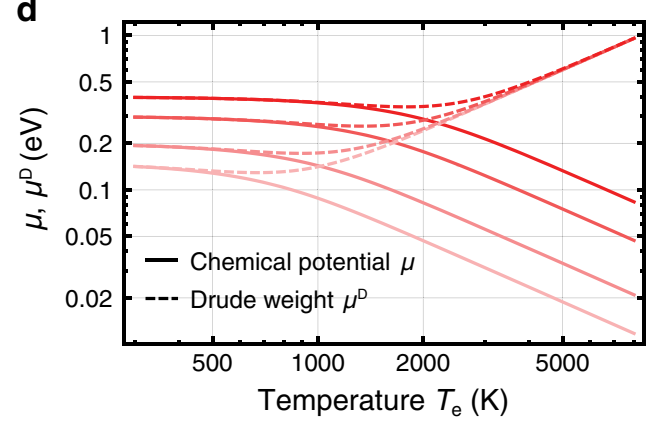

f

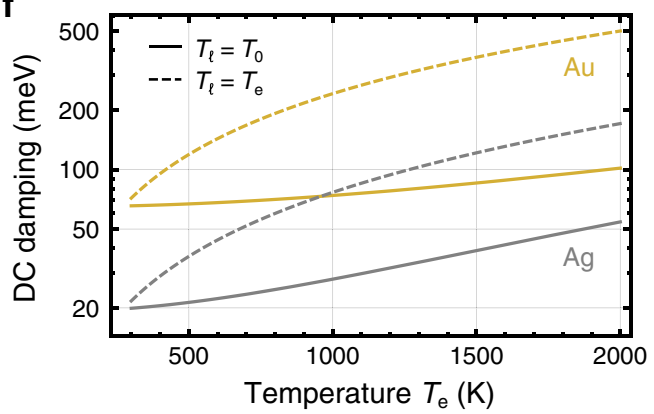

Fig. 1 Thermo-optical properties of graphene and thin metal films. a Schematic representation of a thermoplasmonic light modulation system; a plasmon-supporting structure is optically pumped to generate a high electron temperature in an optical absorber placed inside it, thus inducing sizeable variations in the plasmon frequencies and intensities that are in turn translated into a large modulation of scattered probe light. $\mathbf{b}$ We consider thermoplasmonic systems combining graphene and thin metallic films, in which pumping light is preferentially absorbed by the former; the spectral operation range is indicated for each of these structures. c Dependence of the electronic heat capacity of graphene (calculated from Eq. (4) in Methods for different Fermi energies $E_{\mathrm{F}}$ ) and thin metallic films (obtained from ref. ${ }^{70}$ for different thicknesses $h$ ) on electron temperature $T_{\mathrm{e}}$ when the lattice temperature $T_{\ell}$ remains at the room value $T_{0}=300 \mathrm{~K} \cdot \mathbf{d} T_{\mathrm{e}}$ dependence of the chemical potential $\mu$ (solid curves) and Drude weight $\mu^{\mathrm{D}}$ (dashed curves) in graphene for different Fermi energies (see color code in e). e, $\mathbf{f} T_{\mathrm{e}}$ dependence of the DC scattering rate in $\mathbf{e}$ graphene and f noble metals when the lattice temperature is either $T_{\ell}=T_{0}$ (solid curves) or $T_{\ell}=T_{\mathrm{e}}$ (dashed curves)

optical conductivity $\sigma$, as shown in Eq. (1) of the Methods section. The chemical potential $\mu$ decreases monotonically with increasing $T_{\mathrm{e}}$ at different Fermi levels, while the Drude weight $\mu^{\mathrm{D}}$ increases dramatically after a slight initial decrease as the temperature is raised (see Fig. 1d). The nontrivial temperature dependence of $\mu^{\mathrm{D}}$ defines the resonance position of graphene plasmons, acting as an effective doping from the optical viewpoint (i.e., more low-energy electron transitions become available and contribute to the plasmonic strength as the Fermi level is smeared out with increasing temperature). In contrast, the plasmon frequencies in noble metals exhibit a very mild dependence on the electron temperature (see Methods).
The inelastic scattering rate of graphene also depends strongly on $T_{\mathrm{e}}$, as shown in Fig. 1e within the DC limit for clean samples (see Methods). During the first picosecond after optical pumping, we typically have $T_{\mathrm{e}} \gg T_{\ell}$ and $T_{\ell} \approx T_{0}=300 \mathrm{~K}$; under these conditions, the inelastic decay rate takes the values shown by solid curves in Fig. 1e. Eventually, the electrons and the lattice thermalize to a common temperature $T_{\ell}=T_{\mathrm{e}}$, a situation in which the scattering rate becomes larger as a consequence of stronger electron-phonon coupling (dashed curves in Fig. 1e). We also note that the scattering rate increases with $E_{\mathrm{F}}$ because the phase space for electron-phonon interactions is enlarged. Similar trends of the temperature 
dependence of the scattering rates are found in noble metals, as shown in Fig. If (see Methods).

In Fig. 2, we show the dispersion relations of three types of plasmonic modes supported by the structures sketched in the insets at three different graphene electron temperatures $T_{\mathrm{e}}$, as calculated using methods discussed in previous studies ${ }^{37,41,54}$. In all cases considered in Fig. 2, we find a strong thermoplasmonic modulation, showing that the plasmons become weaker at a higher $T_{\mathrm{e}}$ as a result of the increase in $\operatorname{Re}\{\sigma\}$. For the mid-IR graphene plasmons considered in Fig. 2a, c, the thermoplasmonic modulation can be mainly attributed to an increase in the inelastic scattering rate when $T_{\mathrm{e}}$ rises (see Fig. 1e). We note that these types of plasmons are extremely subwavelength, deviating far away from the light line (blue broken lines, almost indistinguishable from the vertical axis) for increasing plasmon energies. In particular, acoustic plasmons, which are a special case of graphene plasmons that arise when a carbon sheet and a metal are brought in close proximity ${ }^{55,56}$, exhibit dispersion relations that are close to the onset of intraband electron-hole pair excitations in graphene (see Fig. 2c, red broken lines). Notably, the spacer thickness strongly affects the acoustic plasmon dispersion: in general, a thinner spacer produces shifts of the plasmon resonance towards lower frequencies, therefore resulting in more confined plasmons. In particular, spacer thicknesses down to $1 \mathrm{~nm}$ can be achieved using a few layers of exfoliated hexagonal boron nitride $(\mathrm{hBN})$, while even a spacer consisting of a single $\mathrm{hBN}$ layer (roughly $0.4 \mathrm{~nm}$ ) has been reported in the

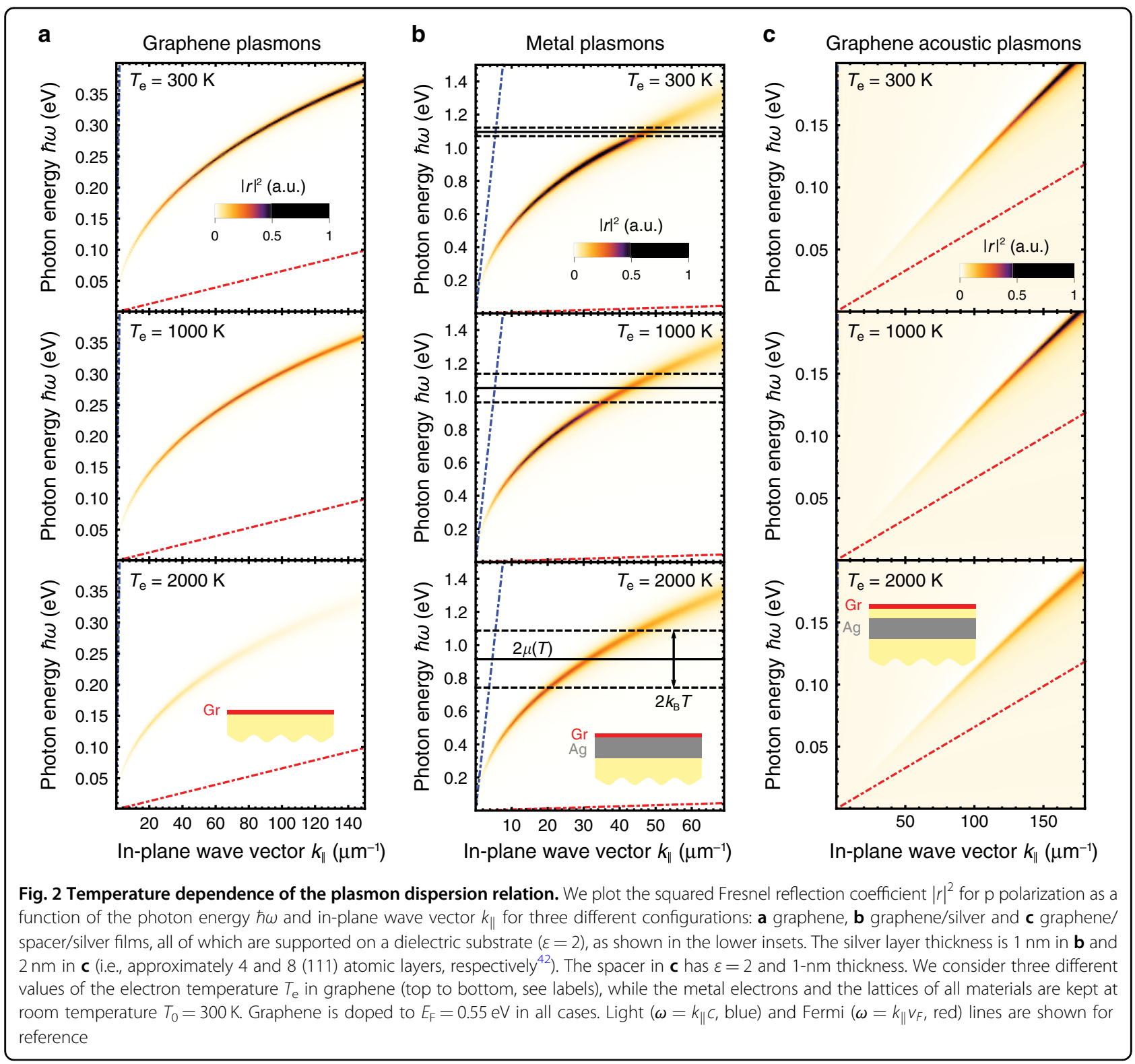


literature ${ }^{56}$. More information regarding the dependence of the acoustic plasmon dispersion on the spacer thickness and Fermi level is presented in Fig. S2 of SI. In contrast, metal plasmons (Fig. 2b), although still very subwavelength, are less confined; at low temperatures (Fig. 2b, top), they are quenched by coupling to interband transitions at photon energies above $2 E_{\mathrm{F}}$ (solid horizontal line), while thermal smearing of Dirac fermions shifts down the chemical potential $\mu$ for the $T_{\mathrm{e}}$ values under consideration and diffuses the quenching within an energy interval of roughly $\pm k_{\mathrm{B}} T_{\mathrm{e}}$ (dashed horizontal lines) around $2 \mu$ (solid horizontal lines).

\section{All-optical manipulation of plasmons in planar structures}

Because plasmon resonances are deep subwavelength excitations (see Fig. 2), extra momentum is required to couple external light into them, typically provided by lateral patterning (e.g., carving gratings or imprinting surface modulations ${ }^{57}$ ) or additional elements (e.g., sharp tips in scanning near-field optical microscopes). Recently, an alternative route to excite graphene plasmons has been proposed $^{49}$, where two interfering optical pump beams create a periodic spatial temperature profile in graphene to assist the coupling of a probe beam to plasmons. The characteristic diffusion time of the temperature profile created by the optical pump is of the order of a few hundred femtoseconds ${ }^{58}$, which should allow a probed optical pulse to excite plasmons, as considered in this study. Here, we further demonstrate that not only graphene plasmons but also metal plasmons or even subwavelength acoustic plasmons can be excited using this approach. We assume a periodic pattern of electron temperature $T_{\mathrm{e}}$ created by the optical grating pump only inside the graphene sheet with a modulated temperature range extending from 300 to $8000 \mathrm{~K}$. Due to the strong temperature dependence of the graphene conductivity $\sigma$ (see Fig. 1), this is spatially modulated and serves as an effective grating (see Fig. 3a). We simulate this type of periodic system using a previously described numerical procedure $^{55}$.

Applying this method to a single extended graphene sheet supported on an $\varepsilon=2$ substrate, we indeed find graphene plasmons in the absorbance spectra of a light probe (Fig. 3b), which are more pronounced for lower Fermi energies due to the stronger spatial variation in the graphene conductivity (see Fig. S3a in SI). The corresponding resonant near-field distribution (Fig. 3e) reveals plasmon confinement around the minima of $T_{\mathrm{e}}$, where Re $\{\sigma\}$ is also minimum (see Fig. S3a). The presence of clear plasmonic resonances even in a pattern with such high temperatures shows that the larger losses experienced by plasmons at higher temperatures are not critical, as long as the lower part of the temperature modulation, where the plasmons are located, is sufficiently deep.
When graphene sits on top of a thin silver film separated by a thin dielectric spacer (see insets in Fig. 3c, d), the hybrid system can support both metal-like and acoustic plasmons. In this configuration, the probing light can still be scattered by the spatial modulation of the graphene conductivity, and therefore, for appropriate photon energies, it can excite those types of plasmons. When exploring the NIR optical response of this hybrid structure, we find absorption peaks associated with metal plasmons, in which the near-field distribution penetrates significantly into the metal film (see Fig. 3f). As a result, we find that $3.36 \%$ and $0.76 \%$ of the probe light are absorbed at the spectral peak B by metal and graphene layers, respectively. In addition, multiple acoustic plasmons can be excited in the same hybrid system in the THz domain, as shown in Fig. 3d. We plot the near-field distributions of the first two of them in Fig. $3 \mathrm{~g}$, where the plasmon energy is observed to be transversally confined inside the dielectric spacer but also laterally concentrated around the regions of minimum $\mathrm{Re}$ $\{\sigma\}$ (see Fig. 3a), with a larger number of nodes along the horizontal direction for higher energy modes, similar to classical standing waves of increasing order in a confining potential. At the first acoustic plasmon resonance (peak C), $15.09 \%$ and $0.33 \%$ of the probe light are absorbed by the metal and graphene, respectively. The larger absorption observed for acoustic plasmons is presumably due to their tighter vertical confinement, which maximizes the fraction of the mode energy present in the metal.

\section{Thermal modulation of ultrathin structured films}

Lateral patterning of the structures considered above directly enables light coupling to their plasmons. In Fig. 4 and S4, we study the thermo-optical properties of these types of structures by considering a thin metal grating placed underneath a graphene sheet. The spectral positions of the plasmons sampled by external light are then controlled by the grating period and metal thickness.

We first investigate metal-like plasmons occurring at a higher energy and supported primarily by the metal. In Fig. 4a, we consider graphene doped to a Fermi energy $E_{\mathrm{F}}=0.55 \mathrm{eV}$ and a silver grating period chosen such that the plasmon energy is slightly smaller than $2 E_{\mathrm{F}}$, so that a large modulation is expected by thermally activating interband transitions within a spectral region of size $\pm k_{\mathrm{B}} T_{\mathrm{e}}$ around $2 E_{\mathrm{F}}$, similar to the results presented in Fig. 2 . At room temperature, $2 \mu \approx 2 E_{\mathrm{F}}$ is above the plasmon energy, so we have well-defined modes with a quality factor $\sim 12.5$ observed in the reflection spectrum of the patterned 1-nm silver film (see $T_{\mathrm{e}}=300 \mathrm{~K}$ curve in Fig. 4a). However, when graphene electrons are heated to a high temperature via ultrafast optical pumping, the chemical potential can be reduced (Fig. 1d), enabling Landau damping of the plasmon, whose quality factor drops to 6.3 at $T_{\mathrm{e}}=5000 \mathrm{~K}$ (see Fig. 4a). In contrast, for a 10-nm-thick 


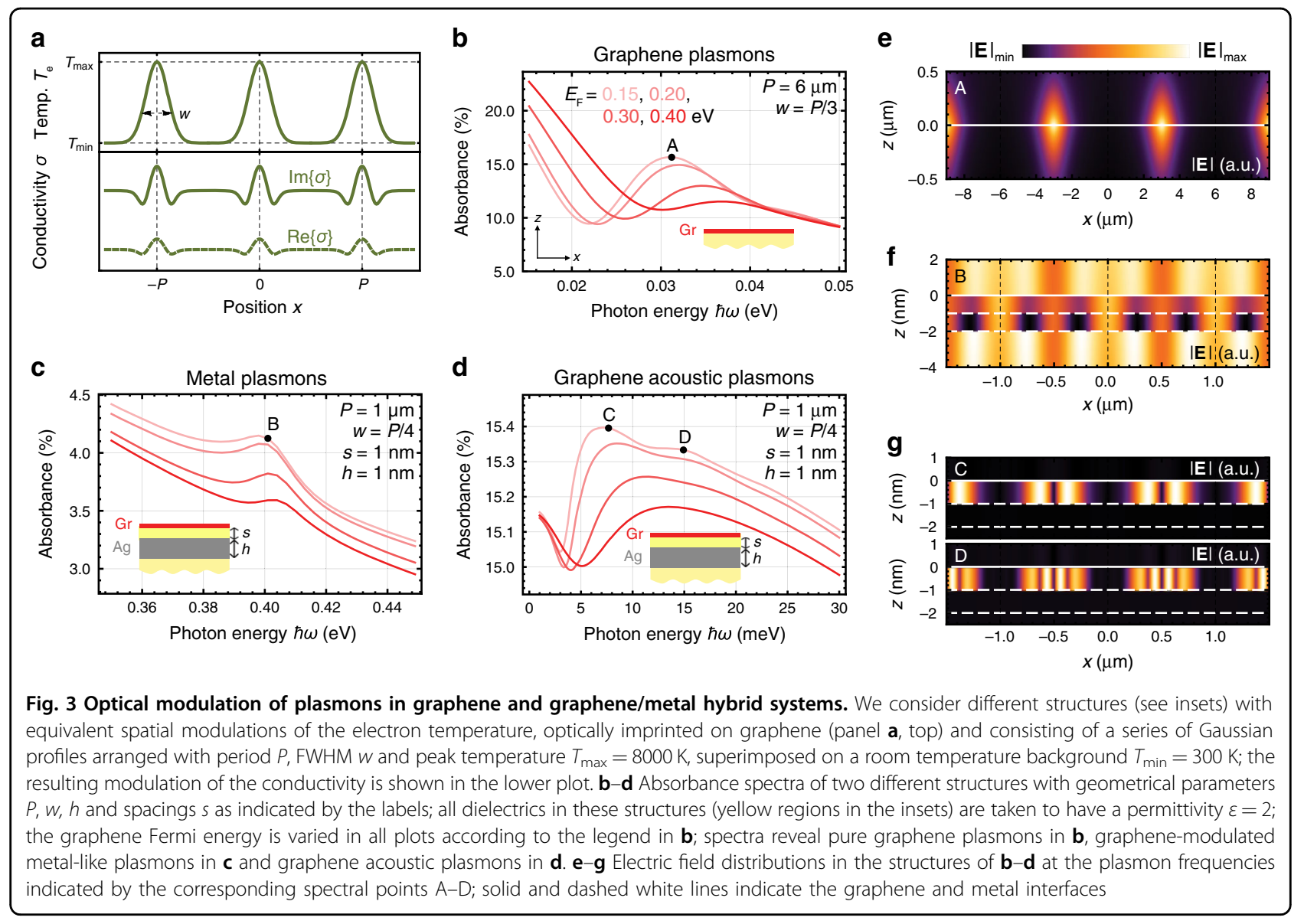

silver ribbon array, plasmons are already substantially broader at room temperature (see Fig. 4c), presumably as a result of larger radiative losses ${ }^{42}$, while we observe weaker thermoplasmonic modulation due in part to the smaller fraction of plasmon energy overlapping the lossy graphene region as the film is made thicker. The peak reflectance at the NIR plasmon resonance of gold and silver gratings in the hybrid structures under consideration is summarized in Fig. 4e as a function of $T_{\mathrm{e}}$ for different metal thicknesses. The modulation depth, which is larger for silver and increases in thinner metal films, takes values as large as $\sim 70 \%$ for the 1 -nm-thick silver structure. These results demonstrate that the thermo-optical response of graphene allows us to reach similar levels of NIR light modulation in neighboring metal plasmons, as previously predicted by exploiting the electro-optical response of this material ${ }^{41}$.

The addition of a 1-nm-thick dielectric spacer between graphene and the thin silver grating enables pronounced acoustic plasmon resonances in the $\mathrm{THz}$ range at $T_{\mathrm{e}}=$ $300 \mathrm{~K}$, where both the first-order and second-order modes are observed in the reflection spectra (Fig. 4b). Similarly, at the elevated electron temperatures induced by optical pumping, those resonances are damped, driving a change in the quality factor from 7.8 at $T_{\mathrm{e}}=300 \mathrm{~K}$ to 2.6 at $T_{\mathrm{e}}=$ $5000 \mathrm{~K}$ in the first-order resonance. In contrast to metal plasmons, the mode energies in this regime are far from the interband transition region, thus preventing Landau damping; plasmon damping is instead mediated by the increase in the inelastic scattering rate with temperature (see Fig. 1e). In addition, the nontrivial spectral shift of the plasmon resonance with varying $T_{\mathrm{e}}$ closely follows the temperature behavior of the Drude weight shown in Fig. 1d. It should be noted that, unlike metal plasmons, there is not a strong dependence on the metal thickness (cf. Fig. 4b, d) because the acoustic plasmon energy is mainly concentrated inside the dielectric spacer (see Fig. 3g), and a 1-nm metal film already produces nearly maximum confinement ${ }^{59}$. In fact, the extended study of the modulation depth of acoustic plasmons presented in Fig. $4 \mathrm{f}$ indicates a remarkable maximum value of $\sim 80 \%$, independent of both thickness and type of metal.

We finally explore in Fig. 5 (see also Fig. S5 in SI) the modulation that can be reached in metallic gratings without any graphene, for which we have to adjust the pump fluence in order to heat the metal electrons to a sufficiently high temperature. Thermo-optical modulation is then produced due to the increase in the inelastic scattering rate with 

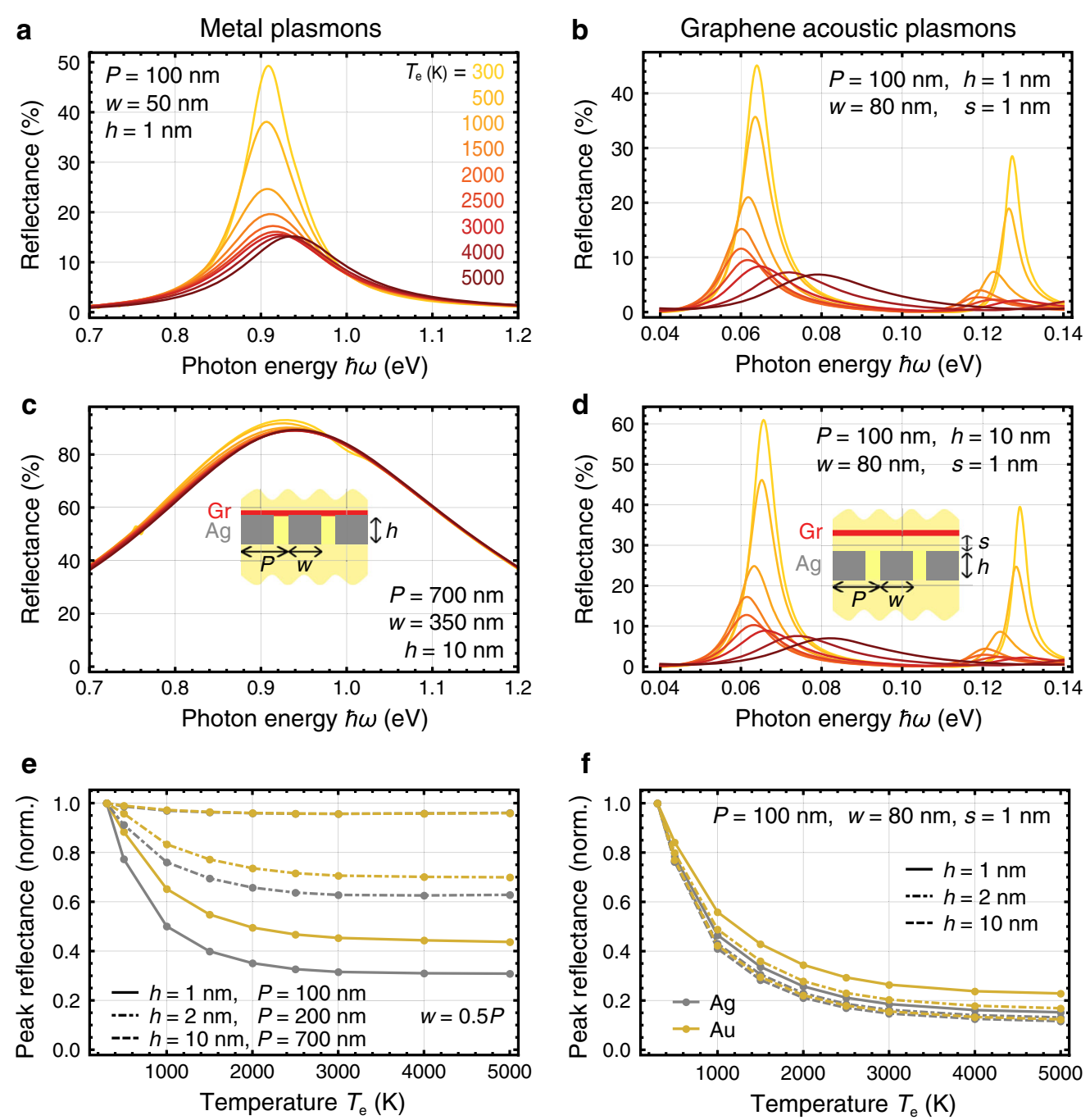

Fig. 4 Thermal modulation of plasmons in hybrid graphene/structured-metal systems. a-d Variation in the reflection spectra of graphene/ silver gratings in the $\mathbf{a}, \mathbf{c} N I R$ and $\mathbf{b}, \mathbf{d}$ far/mid-IR plasmonic regions for different graphene electron temperatures $T_{e}$; the silver thickness is $1 \mathrm{~nm}$ in $\mathbf{a}, \mathbf{b}$ and $10 \mathrm{~nm}$ in $\mathbf{c}, \mathbf{d}$. e, $\mathbf{f}$ Peak reflectance as a function of the graphene electron temperature for structures with different types of metal and metal thicknesses (see geometrical parameters indicated by labels). In $\mathbf{a}, \mathbf{c}, \mathbf{e}$, plasmons are metal-like, and graphene is doped to $E_{\mathrm{F}}=0.55 \mathrm{eV}$. In b, d, f, plasmons are acoustic, and graphene is doped to $E_{\mathrm{F}}=0.4 \mathrm{eV}$. All structures are embedded in an $\varepsilon=2$ dielectri

temperature (see Fig. 1f). The reflection spectra plotted in Fig. 5a show a clear variation in the quality factor for a 1 -nm-thick gold ribbon array as the pump fluence is increased. Specifically, the quality factor drops from 5.3 to 4.0 when the fluence varies in the $0-150 \mathrm{~mJ} / \mathrm{m}^{2}$ range. Because the temperature elevation is smaller in thicker metal structures (see Fig. 5c and Fig. S1 in SI), we expect a weaker temperature dependence in the reflection spectra, which is corroborated in Fig. $5 \mathrm{~b}$ for a thickness of $10 \mathrm{~nm}$. We summarize the relative variation in the peak reflectance as a function of pump fluence for different materials and metal thicknesses in Fig. 5d. In contrast to the hybrid graphene/ metal structures, we now observe a larger modulation with gold than with silver. In particular, a maximum modulation depth of $\sim 10 \%(\sim 30 \%)$ is predicted for a pump fluence of $20 \mathrm{~mJ} / \mathrm{m}^{2}\left(100 \mathrm{~mJ} / \mathrm{m}^{2}\right)$ in 1-nm-thick (four atomic layers) gold gratings, which is a smaller value than in hybrid structures, despite the large fluences under consideration compared with those needed to heat graphene electrons to the values of $T_{\mathrm{e}}$ used in Fig. 4.

\section{Discussion}

In summary, the remarkably small electronic heat capacity of atomically thin systems, such as graphene and few-atomic-layer noble metal films, allows us to elevate their electron temperature via ultrafast optical pumping in such a way that their optical responses are dramatically modified with moderate pump intensities below the 

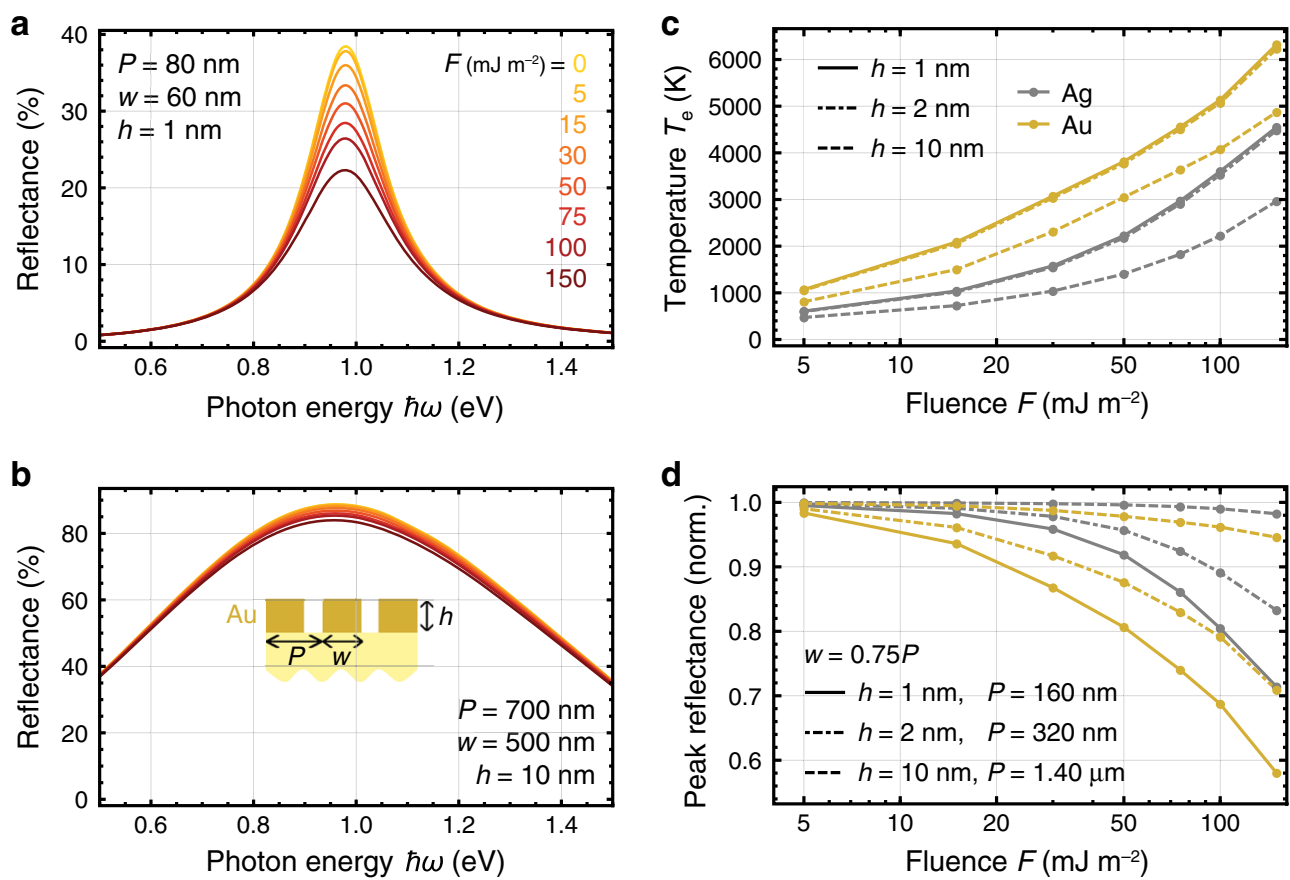

Fig. 5 Thermal modulation of metal plasmons. a, b Variation in the probed reflection spectra in a gold ribbon array (see inset in $\mathbf{b}$ ) under different pump fluences at $785 \mathrm{~nm}$ wavelength; the gold thickness is $1 \mathrm{~nm}$ in $\mathbf{a}$ and $10 \mathrm{~nm}$ in $\mathbf{b}$. c Fluence dependence of the electron temperature of extended gold and silver films of different thicknesses immediately after optical pumping. $\mathbf{d}$ Peak reflectance as a function of the pump fluence for different metal types and thicknesses, normalized to the value at zero fluence with geometrical parameters as indicated by labels. The ribbon array is placed on an $\varepsilon=2$ dielectric substate in all cases

damage threshold. This effect, which constitutes the basis for ultrafast thermo-optical modulation, is particularly strong in graphene due to the large photothermal response of this material, in which the conical electronic band structure causes large changes in the optical behavior for moderate amounts of deposited electronic heat. The photothermal modulation of the frequency and strength of graphene plasmons has been extensively studied in the past ${ }^{37}$, but their applicability has been limited to the mid-IR regime. We have shown that the addition of a noble metal film in close proximity to graphene enables similar levels of modulation $>70 \%$ to be reached over a wider spectral range extending down to the visible domain. More precisely, optical pumping can bring graphene electrons to a high temperature, while the large heat capacity of the metal prevents similar heating inside it; then, high-energy metal-like plasmons are strongly affected by the opening of additional loss channels (i.e., formerly forbidden electron-hole pair transitions in the hot graphene electron gas); additionally, lower-frequency acoustic plasmons supported in these hybrid structures also become more lossy. A practical way to exploit these effects consists in laterally patterning the metal (e.g., into ribbons), thus breaking the energy-momentum mismatch that otherwise prevents light coupling into the plasmons of planar films. We envision applications in ultrafast optical modulation, which could be potentially pushed to the single-photon level through plasmon blockade in sufficiently small graphene nanostructures ${ }^{60}$. Spectrally resolved optical sensing could also be carried out by exploiting the temperature-dependent plasmon shifts reported here, which would enable a continuous sweep of the probe frequency as the system cools down over a picosecond timescale. Although the achieved thermallyinduced spectral shifts can be limited by the presence of a finite diffusion time in the temperature profile, the amplitudes of the associated changes in the reflectivity provide an alternative sizeable signature for optical sensing in the $\sim 300-3000 \mathrm{~K}$ temperature range.

\section{Methods}

\section{Graphene conductivity}

We use the local random-phase approximation to calculate the optical conductivity of graphene as ${ }^{61,62}$

$\sigma(\omega)=\frac{e^{2}}{\pi \hbar^{2}} \frac{\mathrm{i}}{\omega+\mathrm{i} / \tau}\left[\mu^{\mathrm{D}}-\int_{0}^{\infty} \mathrm{d} E \frac{f_{\mu, T_{\mathrm{e}}}(E)-f_{\mu, T_{\mathrm{e}}}(-E)}{1-4 E^{2} /\left[\hbar^{2}(\omega+\mathrm{i} / \tau)^{2}\right]}\right]$

where $\tau$ is a phenomenological inelastic scattering lifetime, $f_{\mu, T_{\mathrm{e}}}(E)=\left[\mathrm{e}^{(E-\mu) / k_{\mathrm{B}} T_{\mathrm{e}}}+1\right]^{-1}$ is the Fermi-Dirac 
Table 1 Parameters used in Eqs. (6), (7) and (8) to calculate the temperature-dependent dielectric function of gold and silver, along with source references

\begin{tabular}{lllllllll}
\hline Metal & $\varepsilon_{\infty}{ }^{69}$ & $\hbar \omega_{\mathrm{p}}\left(T_{0}\right)(\mathrm{eV})^{69}$ & $B\left(\mathrm{~K}^{-1}\right)^{71}$ & $E_{\mathrm{F}}(\mathrm{eV})^{46}$ & $\Gamma^{66}$ & $\Delta^{66}$ & $\theta_{\mathrm{D}}(\mathrm{K})^{72}$ & $\hbar \gamma_{\mathrm{o}}(\mathrm{meV})$ \\
\hline $\mathrm{Au}$ & 9.5 & 9.06 & $14.2 \times 10^{-6}$ & 5.53 & 0.55 & 0.77 & 170 & 39.5 \\
$\mathrm{Ag}$ & 4.0 & 9.17 & $19.7 \times 10^{-6}$ & 5.49 & 0.55 & 0.73 & 215 & 14.5 \\
\hline
\end{tabular}

distribution at an electron temperature $T_{\mathrm{e}}$, energy $E$ and chemical potential $\mu, k_{\mathrm{B}}$ is the Boltzmann constant and $\mu^{\mathrm{D}}=\mu+2 k_{\mathrm{B}} T_{\mathrm{e}} \log \left(1+\mathrm{e}^{-\mu / k_{\mathrm{B}} T_{\mathrm{e}}}\right)$ is the Drude weight. The temperature-corrected chemical potential can be well approximated by ${ }^{63}$

$$
\mu \approx \sqrt{\sqrt{E_{\mathrm{F}}^{4}+\left(2 \log ^{2} 4\right)^{2}\left(k_{\mathrm{B}} T_{\mathrm{e}}\right)^{4}}-2 \log ^{2} 4\left(k_{\mathrm{B}} T_{\mathrm{e}}\right)^{2}}
$$

where $E_{\mathrm{F}}$ is the Fermi energy defined at zero temperature. The graphene conductivity has two contributions corresponding to the electronic intraband (first term in Eq. (1), proportional to $\mu^{\mathrm{D}}$ ) and interband (integral term in Eq. (1)) transitions. Pauli blocking forbids interband transitions with energy below $2 E_{\mathrm{F}}$ at zero temperature, implying that the intraband response dominates at low temperatures and photon energies, where $\operatorname{Re}\{\sigma\}$ is roughly proportional to $1 / \tau$ (i.e., the intrinsic optical losses). However, at higher electron temperatures, the chemical potential becomes increasingly smaller than $E_{\mathrm{F}}$ (Fig. 1d), allowing transitions to take place at increasingly lower energies and therefore enhancing $\operatorname{Re}\{\sigma\}$.

In general, the inelastic scattering rate $1 / \tau$ depends on both electron and lattice temperatures. For clean graphene samples (e.g., encapsulated graphene), the inelastic scattering rate is dominated by electron-phonon scattering. Following previous studies ${ }^{64,65}$, the energy-dependent scattering rate associated with the $b$-type phonon is given to first order by

$$
\frac{1}{\tau_{b}\left(\varepsilon_{\mathbf{k}}^{l}\right)}=\sum_{\mathbf{k}^{\prime}, l^{\prime}} P_{\mathbf{k} \mathbf{k}^{\prime}, b}^{l l^{\prime}} \frac{1-f_{\mu, T_{\mathrm{e}}}\left(E_{\mathbf{k}^{\prime}}^{l^{\prime}}\right)}{1-f_{\mu, T_{\mathrm{e}}}\left(E_{\mathbf{k}}^{l}\right)}\left(1-\cos \theta_{\mathbf{k k} /}\right)
$$

where $\theta_{\mathbf{k} \mathbf{k}^{\prime}}$ is the scattering angle between incoming and outgoing wave vectors $\mathbf{k}$ and $\mathbf{k}^{\prime}, E_{\mathbf{k}}^{l}=l \hbar v_{\mathrm{F}}|\mathbf{k}|$ denotes the electron $(l=1)$ and hole $(l=-1)$ energies in the conical band dispersion regime, $v_{\mathrm{F}} \approx 10^{6} \mathrm{~ms}^{-1}$ is the Fermi velocity and

$P_{\mathbf{k k}^{\prime}, b}^{l l^{\prime}}=\frac{2 \pi}{\hbar}\left|g_{\mathbf{k} \mathbf{k}^{\prime}, b}^{l l^{\prime}}\right|^{2}\left\{\begin{array}{l}n\left(\omega_{\mathbf{q}, b}\right) \delta\left(E_{\mathbf{k}^{\prime}}^{l^{\prime}}-E_{\mathbf{k}}^{l}-\hbar \omega_{\mathbf{q}, b}\right) \\ +\left[n\left(\omega_{\mathbf{q}, b}\right)+1\right] \delta\left(E_{\mathbf{k}^{\prime}}^{l^{\prime}}-E_{\mathbf{k}}^{l}+\hbar \omega_{\mathbf{q}, b}\right)\end{array}\right\}$

is the electron- $b$-phonon scattering probability. Here, $\mathbf{q}=$ $\mathbf{k}^{\prime}-\mathbf{k}, n(\omega)=\left[\mathrm{e}^{\hbar \omega / k_{\mathrm{B}} T_{\ell}}-1\right]^{-1}$ is the Bose-Einstein distribution, $T_{\ell}$ is the lattice temperature and $g_{\mathbf{k k}^{\prime}, b}^{l l^{\prime}}$ are matrix elements for the $b$-type phonon that can be readily evaluated using Eqs. (16-21) and Table IV of ref. ${ }^{64}$. Then, the total DC inelastic scattering rate is given by

$$
\frac{1}{\tau}=\frac{\int \mathrm{d} E D(E) \partial f_{\mu, T_{\mathrm{e}}}(E) / \partial E}{\int \mathrm{d} E D(E)\left[\sum_{b} \tau_{b}^{-1}(E)\right]^{-1} \partial f_{\mu, T_{\mathrm{e}}}(E) / \partial E}
$$

where $D(E)=2|E| / \pi \hbar^{2} v_{\mathrm{F}}^{2}$ is the electron density of states in graphene, including spin and valley degeneracy. We present in Fig. 1e the total inelastic scattering rate obtained from Eq. (3).

\section{Graphene heat capacity}

The specific heat capacity of graphene (Fig. 1c) is calculated from the slope of the $T_{\mathrm{e}}$-dependent electronic heat as ${ }^{63}$

$$
c_{\mathrm{e}}=\frac{\partial}{\partial T_{\mathrm{e}}}\left[\beta \frac{\left(k_{\mathrm{B}} T_{\mathrm{e}}\right)^{3}}{\left(\hbar v_{\mathrm{F}}\right)^{2}}\right]
$$

where

$$
\beta=\frac{2}{\pi}\left[\int_{0}^{\infty} x^{2} \mathrm{~d} x\left(\frac{1}{\mathrm{e}^{x+\mu / k_{\mathrm{B}} T_{\mathrm{e}}}+1}+\frac{1}{\mathrm{e}^{x-\mu / k_{\mathrm{B}} T_{\mathrm{e}}}+1}\right)-\frac{1}{3}\left(\frac{E_{\mathrm{F}}}{k_{\mathrm{B}} T_{\mathrm{e}}}\right)^{3}\right]
$$

is a thermal coefficient.

\section{Temperature-dependent permittivity of noble metals}

For ultrathin metal films, a recent study shows that the Drude response works extremely well compared with the random-phase approximation incorporating film electron wave functions and atomic-plane corrugation ${ }^{59}$. In addition, the Drude model is in agreement with recent observations of plasmons supported by few-atomic-layer crystalline silver films ${ }^{42}$. We therefore use the Drude model and write the metal permittivity as ${ }^{48}$

$$
\epsilon_{\mathrm{m}}\left(\omega, T_{\mathrm{e}}, T_{\ell}\right)=\epsilon_{\infty}-\frac{\omega_{\mathrm{p}}^{2}\left(T_{\ell}\right)}{\omega\left[\omega+\mathrm{i} \gamma_{\mathrm{m}}\left(T_{\mathrm{e}}, T_{\ell}\right)\right]}
$$

where $\varepsilon_{\infty}$ is a background accounting for the polarization of interband transitions, the plasmon frequency $\omega_{\mathrm{p}}\left(T_{\ell}\right)=\omega_{\mathrm{p}}\left(T_{0}\right) / \sqrt{1+B\left(T_{\ell}-T_{0}\right)}$ depends on lattice temperature $T_{\ell}$ through the thermal expansion coefficient 
$B$ (see Table 1), and the phenomenological damping rate $\gamma_{\mathrm{m}}\left(T_{\mathrm{e}}, T_{\ell}\right)=\gamma_{\mathrm{m}}^{\mathrm{e}-\mathrm{e}}\left(T_{\mathrm{e}}\right)+\gamma_{\mathrm{m}}^{\mathrm{e}-\mathrm{ph}}\left(T_{\ell}\right)$ is the sum of the temperature-dependent electron-electron and electronphonon contributions. More precisely ${ }^{66-68}$,

$$
\gamma_{\mathrm{m}}^{\mathrm{e}-\mathrm{e}}\left(T_{\mathrm{e}}\right)=\frac{\pi^{3} \Gamma \Delta}{12 \hbar E_{\mathrm{F}}} k_{\mathrm{B}}^{2} T_{\mathrm{e}}^{2}
$$

(see Table 1 for values of the Fermi energy $E_{\mathrm{F}}$ and scattering coefficients $\Gamma$ and $\Delta$ ) and ${ }^{67,68}$

$$
\gamma_{\mathrm{m}}^{\mathrm{e}-\mathrm{ph}}\left(T_{\ell}\right)=\gamma_{0}\left(\frac{2}{5}+\frac{4 T_{\ell}^{5}}{\theta_{\mathrm{D}}^{5}} \int_{0}^{\theta_{\mathrm{D}} / T_{\ell}} \frac{z^{4} d z}{\mathrm{e}^{z}-1}\right) \approx \gamma_{0} \frac{T_{\ell}}{\theta_{\mathrm{D}}}
$$

where $\gamma_{0}$ is found by matching the total inelastic scattering rate $\gamma_{\mathrm{m}}\left(T_{0}, T_{0}\right)$ to the experimental results at ambient temperature $T_{0}=300 \mathrm{~K}$ (i.e., $\hbar \gamma_{\mathrm{m}}=71 \mathrm{meV}$ and $21 \mathrm{meV}$ for gold and silver, respectively ${ }^{69}$; see Table 1 for the values of $\gamma_{0}$ and the Debye temperature $\theta_{\mathrm{D}}$ ). The rightmost approximate expression in Eq. (8) is valid in the $T_{\ell} \gg \theta_{\mathrm{D}}$ limit. We plot $\gamma_{\mathrm{m}}$ in Fig. If for Au and Ag with different temperature combinations. We further use Eq. (6) to calculate the optical spectra shown in Fig. 5. We emphasize that the $T_{\mathrm{e}}$ dependence of the plasmon resonance frequency of metallic nanostructures is mostly controlled by that of the classical metal plasma frequency $\omega_{\mathrm{p}}$, which is robust against the electron temperature range considered in this work (see Fig. 5 and Fig. S5 in SI).

\section{Acknowledgements}

This work has been supported in part by the ERC (Advanced Grant 789104eNANO), the Spanish MINECO (MAT2017-88492-R and SEV2015-0522), the Catalan CERCA Program, and Fundació Privada Cellex. E.J.C.D. acknowledges financial support from "la Caixa" INPhINIT (Fellowship Grant 1000110434, LCF/ BQ/DI17/11620057) and the EU (Marie Sklodowska-Curie Grant 713673).

\section{Conflict of interest}

The authors declare that they have no conflict of interest.

Supplementary information is available for this paper at https://doi.org/ 10.1038/s41377-020-0322-z.

Received: 12 December 2019 Revised: 20 April 2020 Accepted: 25 April 2020

Published online: 18 May 2020

\section{References}

1. Gobin, A. M. et al. Near-infrared resonant nanoshells for combined optical imaging and photothermal cancer therapy. Nano Lett. 7, 1929-1934 (2007).

2. Jain, P. K., El-Sayed, I. H. \& El-Sayed, M. A. Au nanoparticles target cancer. Nano Today 2, 18-29 (2007).

3. Zhao, W. \& Karp, J. M. Tumour targeting: nanoantennas heat up. Nat. Mater. 8 453-454 (2009).

4. Boyer, D. et al. Photothermal imaging of nanometer-sized metal particles among scatterers. Science 297, 1160-1163 (2002).

5. Berciaud, S. et al. Photothermal heterodyne imaging of individual metallic nanoparticles: theory versus experiment. Phys. Rev. B 73, 045424 (2006).
6. Wang, L. \& Li, B. Thermal memory: a storage of phononic information. Phys. Rev. Lett. 101, 267203 (2008)

7. Pollock, H. M. \& Hammiche, A. Micro-thermal analysis: techniques and applications. J. Phy. D 34, R23-R53 (2001).

8. Yu, R. \& García de Abajo, F. J. Electrical detection of single graphene plasmons. ACS Nano 10,8045-8053 (2016).

9. Guo, Q. et al. Efficient electrical detection of mid-infrared graphene plasmons at room temperature. Nat. Mater. 17, 986-992 (2018).

10. Baffou, G., Quidant, R. \& García de Abajo, F. J. Nanoscale control of optical heating in complex plasmonic systems. ACS Nano 4, 709-716 (2010).

11. Baffou, G. Thermoplasmonics: Heating Metal Nanoparticles Using Light. (Cambridge University Press, Cambridge, 2017).

12. Meng, L. et al. Plasmonic nano-oven by concatenation of multishell photothermal enhancement. ACS Nano 11, 7915-7924 (2017).

13. Thongrattanasiri, S. \& García de Abajo, F. J. Optical field enhancement by strong plasmon interaction in graphene nanostructures. Phys. Rev. Lett. 110, 187401 (2013).

14. Woessner, A. et al. Highly confined low-loss plasmons in graphene-boron nitride heterostructures. Nat. Mater. 14, 421-425 (2015).

15. Lundeberg, M. B. et al. Tuning quantum nonlocal effects in graphene plasmonics. Science 357, 187-191 (2017).

16. $\mathbf{N i}, \mathrm{G}$. X. et al. Fundamental limits to graphene plasmonics. Nature $\mathbf{5 5 7}$ 530-533 (2018).

17. Ju, L. et al. Graphene plasmonics for tunable terahertz metamaterials. Nat. Nanotechnol. 6, 630-634 (2011).

18. Fei, Z. et al. Infrared nanoscopy of dirac plasmons at the graphene-SiO interface. Nano Lett. 11, 4701-4705 (2011).

19. Shin, S. Y. et al. Control of the $\pi$ plasmon in a single layer graphene by charge doping. App. Phys. Lett. 99, 082110 (2011).

20. Fei, Z. et al. Gate-tuning of graphene plasmons revealed by infrared nanoimaging. Nature 487, 82-85 (2012).

21. Chen, J. et al. Optical nano-imaging of gate-tunable graphene plasmons. Nature 487, 77-81 (2012).

22. Yan, $\mathrm{H}$. et al. Tunable infrared plasmonic devices using graphene/insulator stacks. Nat. Nanotechnol. 7, 330-334 (2012).

23. Brar, V. W. et al. Highly confined tunable mid-infrared plasmonics in graphene nanoresonators. Nano Lett. 13, 2541-2547 (2013).

24. Yan, $\mathrm{H}$. et al. Damping pathways of mid-infrared plasmons in graphene nanostructures. Nat. Photon. 7, 394-399 (2013).

25. Shi, $\mathrm{C}$. et al. Tunable terahertz hybrid graphene-metal patterns metamaterials. Optics Laser Technol. 114, 28-34 (2019).

26. He, X. et al. Investigation of terahertz all-dielectric metamaterials. Optics Express 27, 13831-13844 (2019).

27. He, X. et al. Investigation of phonon scattering on the tunable mechanisms of terahertz graphene metamaterials. Nanomaterials 10, E39 (2020).

28. Thongrattanasiri, S. \& Koppens, F. H. L., García de Abajo, F. J. Complete optical absorption in periodically patterned graphene. Phys. Rev. Lett. 108, 047401 (2012).

29. Alaee, R. et al. A perfect absorber made of a graphene micro-ribbon metamaterial. Optics Express 20, 28017-28024 (2012).

30. Jang, M. S. et al. Tunable large resonant absorption in a midinfrared graphene Salisbury screen. Phys. Rev. B 90, 165409 (2014).

31. Kim, S. et al. Electronically tunable perfect absorption in graphene. Nano Lett. 18, 971-979 (2018)

32. Freitag, $M$. et al. Photocurrent in graphene harnessed by tunable intrinsic plasmons. Nat. Commun. 4, 1951 (2013).

33. Luxmoore, I. J. et al. Graphene-metamaterial photodetectors for integrated infrared sensing. ACS Photon. 3, 936-941 (2016).

34. Rodrigo, D. et al. Mid-infrared plasmonic biosensing with graphene. Science 349, 165-168 (2015).

35. Yu, R. et al. Nonlinear plasmonic sensing with nanographene. Phys. Rev. Lett. 117, 123904 (2016).

36. $\mathrm{Hu}, \mathrm{H}$. et al. Far-field nanoscale infrared spectroscopy of vibrational fingerprints of molecules with graphene plasmons. Nat. Commun. 7, 12334 (2016).

37. García de Abajo, F. J. Graphene plasmonics: challenges and opportunities. ACS Photon. 1, 135-152 (2014).

38. Ni, G. X. et al. Ultrafast optical switching of infrared plasmon polaritons in highmobility graphene. Nat. Photon. 10, 244-247 (2016).

39. Jadidi, M. M. et al. Nonlinear terahertz absorption of graphene plasmons. Nano Lett. 16, 2734-2738 (2016).

40. Manjavacas, A. \& García de Abajo, F. J. Tunable plasmons in atomically thin gold nanodisks. Nat. Commun. 5, 3548 (2014). 
41. Yu, R. \& Pruneri, V., García de Abajo, F. J. Active modulation of visible light with graphene-loaded ultrathin metal plasmonic antennas. Sci. Rep. 6, 32144 (2016).

42. El-Fattah, Z. M. A. et al. Plasmonics in atomically thin crystalline silver films. ACS Nano 13, 7771-7779 (2019).

43. Maniyara, R. A. et al. Tunable plasmons in ultrathin metal films. Nat. Photon. 13, 328-333 (2019).

44. Saavedra, J. R. M., Asenjo-Garcia, A. \& García de Abajo, F. J. Hot-electron dynamics and thermalization in small metallic nanoparticles. ACS Photon. 3, 1637-1646 (2016)

45. Efetov, D. K. et al. Fast thermal relaxation in cavity-coupled graphene bolometers with a Johnson noise read-out. Nat. Nanotechnol. 13, 797-801 (2018).

46. Rogalski, M. S. \& Palmer, S. B. Solid State Physics. (CRC Press, London, 2000).

47. Lui, C. H. et al. Ultrafast photoluminescence from graphene. Phys. Rev. Lett. 105, 127404 (2010)

48. Block, A. et al. Tracking ultrafast hot-electron diffusion in space and time by ultrafast thermomodulation microscopy. Sci. Adv. 5, eaav8965 (2019).

49. Yu, R. et al. Photothermal engineering of graphene plasmons. Phys. Rev. Lett. 121, 057404 (2018)

50. Pop, E., Varshney, V. \& Roy, A. K. Thermal properties of graphene: fundamentals and applications. MRS Bulletin 37, 1273-1281 (2012).

51. Johannsen, J. C. et al. Direct view of hot carrier dynamics in graphene. Phys. Rev. Lett. 111, 027403 (2013).

52. Gierz, I. et al. Snapshots of non-equilibrium Dirac carrier distributions in graphene. Nat. Mater. 12, 1119-1124 (2013).

53. Baudisch, M. et al. Ultrafast nonlinear optical response of Dirac fermions in graphene. Nat. Commun. 9, 1018 (2018).

54. Dias, E. J. C. \& García de Abajo, F. J. Fundamental limits to the coupling between light and 2D polaritons by small scatterers. ACS Nano 13, 5184-5197 (2019).

55. Gonçalves, P. A. D. \& Peres, N. M. R. An Introduction to Graphene Plasmonics. (World Scientific, New Jersey, 2016).

56. Iranzo, D. A. et al. Probing the ultimate plasmon confinement limits with a van der Waals heterostructure. Science 360, 291-295 (2018).
57. Farhat, M., Guenneau, S. \& Bağcı, H. Exciting graphene surface plasmon polaritons through light and sound interplay. Phys. Rev. Lett. 111, 237404 (2013).

58. Ruzicka, B. A. et al. Hot carrier diffusion in graphene. Phys. Rev. B 82, 195414 (2010).

59. Rodríguez Echarri, A., Cox, J. D. \& García de Abajo, F. J. Quantum effects in the acoustic plasmons of atomically thin heterostructures. Optica 6, 798 (2019).

60. Cox, J. D. \& García de Abajo, F. J. Single-plasmon thermo-optical switching in graphene. Nano Lett. 19, 3743-3750 (2019).

61. Falkovsky, L. A. \& Varlamov, A. A. Space-time dispersion of graphene conductivity. Eur. Phys. J. B 56, 281-284 (2007).

62. Falkovsky, L. A. \& Pershoguba, S. S. Optical far-infrared properties of a graphene monolayer and multilayer. Phys. Rev. B 76, 153410 (2007).

63. Yu, R., Manjavacas, A. \& García de Abajo, F. J. Ultrafast radiative heat transfer. Nat. Commun. 8, 2 (2017).

64. Sohier, T. et al. Phonon-limited resistivity of graphene by first-principles calculations: electron-phonon interactions, strain-induced gauge field, and Boltzmann equation. Phys. Rev. B 90, 125414 (2014).

65. Yuan, S. et al. Room temperature graphene mid-infrared bolometer with a broad operational wavelength range. ACS Photon. ASAP Articles (2020).

66. Lawrence, W. E. Electron-electron scattering in the low-temperature resistivity of the noble metals. Phys. Rev. B 13, 5316-5319 (1976).

67. McKay, J. A. \& Rayne, J. A. Temperature dependence of the infrared absorptivity of the noble metals. Phys. Rev. B 13, 673-685 (1976).

68. Beach, R. T. \& Christy, R. W. Electron-electron scattering in the intraband optical conductivity of Cu, Ag, and Au. Phys. Rev. B 16, 5277-5284 (1977).

69. Johnson, P. B. \& Christy, R. W. Optical constants of the noble metals. Phys. Rev. B 6, 4370-4379 (1972).

70. Lin, Z., Zhigilei, L. V. \& Celli, V. Electron-phonon coupling and electron heat capacity of metals under conditions of strong electron-phonon nonequilibrium. Phys. Rev. B 77, 075133 (2008).

71. Davis, J. R. Metals Handbook Desk Edition. 2nd edn. (ASM International, Materials Park, Ohio, 1998).

72. O'Reilly, E. Quantum Theory of Solids. 1st edn. (CRC Press, London, 2017) 